\title{
Correlates of Neutralizing/SARS-CoV-2-S1-binding Antibody Response with Adverse Effects and Immune Kinetics in BNT162b2-Vaccinated Individuals
}

Kenji Maeda ( $\boldsymbol{\nabla}$ kmaeda@ri.ncgm.go.jp )

National Center for Global Health and Medicine (NCGM) Research Institute

Masayuki Amano

Kumamoto University Hospital

Yukari Uemura

National Center For Global Health and Medicine

Kiyoto Tsuchiya

AIDS Clinical Center, NCGM

Tomoko Matsushima

Sysmex Corporation

Kenta Noda

Sysmex Corporation

Yosuke Shimizu

National Center For Global Health and Medicine

Asuka Fujiwara

National Center for Global Health and Medicine (NCGM) Research Institute

Yuki Takamatsu

National Center for Global Health and Medicine (NCGM) Research Institute

Yasuko Ichikawa

JCHO Kumamoto General Hospital

Hidehiro Nishimura

JCHO Kumamoto General Hospital

Mari Kinoshita

JCHO Kumamoto General Hospital

Shota Matsumoto

JCHO Kumamoto General Hospital

Hiroyuki Gatanaga

AIDS Clinical Center, NCGM

Kazuhisa Yoshimura

Tokyo Metropolitan Institute for Public Health 


\section{Shinichi Oka}

AIDS Clinical Center, NCGM

\section{Ayako Mikami}

National Center For Global Health and Medicine

\section{Wataru Sugiura}

National Center For Global Health and Medicine

\section{Toshiyuki Sato}

Sysmex Corporation

\section{Tomokazu Yoshida}

Sysmex Corporation

\section{Shinya Shimada}

JCHO Kumamoto General Hospital

\section{Hiroaki Mitsuya}

National Center for Global Health and Medicine (NCGM) Research Institute

\section{Research Article}

Keywords: COVID-19, vaccine, BNT162b2, SARS-CoV-2, neutralizing antibody

Posted Date: September 3rd, 2021

DOl: https://doi.org/10.21203/rs.3.rs-847396/v1

License: (c) (i) This work is licensed under a Creative Commons Attribution 4.0 International License. Read Full License

Version of Record: A version of this preprint was published at Scientific Reports on November 24th, 2021. See the published version at https://doi.org/10.1038/s41598-021-01930-y. 


\section{Abstract}

While mRNA vaccines against SARS-CoV-2 are exceedingly effective in preventing symptomatic infection, their immune response features remain to be clarified. In the present prospective study, 225 healthy individuals in Japan, who received two BNT162b2 doses, were enrolled. Correlates of BNT162b2-elicited SARS-CoV-2-neutralizing activity ( $50 \%$ neutralization titer: $\mathrm{NT}_{50}$; assessed using infectious virions) with various determinants were examined and the potency of serums against variants of concerns was determined. Significant rise in $\mathrm{NT}_{50} \mathrm{~s}$ was seen in serums on day 28 post- 1 st dose. A moderate inverse correlation was seen between $\mathrm{NT}_{50} \mathrm{~s}$ and ages, but no correlation seen between $\mathrm{NT}_{50} \mathrm{~S}$ and adverse effects. $\mathrm{NT}_{50}$ s and SARS-CoV-2-S1-binding-lgG levels on day 28 post-1 st dose and pain scores following the 2 nd shot were greater in women than in men. The average half-life of $\mathrm{NT}_{50} \mathrm{~s}$ was $\sim 68$ days and the estimated average time length till the total disappearance of neutralizing activity was $\sim 198$ days. While serums from elite-responders ( $\mathrm{NT}_{50} \mathrm{~s}>1,500$-fold: the top $4 \%$ among the participants) potently to moderately blocked all variants of concerns examined, some serums with low $\mathrm{NT}_{50} \mathrm{~s}$ failed to block the B.1.351-beta strain. Since BNT162b2-elicited immunity against SARS-CoV-2 is short, an additional vaccine or other protective measures are needed.

\section{Introduction}

Since the emergence of coronavirus disease 2019 (COVID-19) caused by severe acute respiratory syndrome coronavirus 2 (SARS-CoV-2) in Wuhan, China, the disease quickly spread to the world. As of June 29, 2021, more than 180 million SARS-CoV-2-infected individuals and almost 4 million death cases have been reported in over 200 countries $^{1-4}$. Since the beginning of the pandemic, researchers and pharmaceutical companies around the world have been working on developing vaccines ${ }^{5}$. Currently, more than 10 vaccines have been authorized for public use worldwide. The development of vaccines against SARS-CoV-2 was achieved time- and efficacy-wise beyond our expectations within a single calendar year from the availability of the viral sequence to the initiation of immunization of many people in several countries ${ }^{6,7}$.

Among various vaccines, two RNA vaccines (BNT162b2 and mRNA-1273/TAK-919) have been shown to be as much as $94-95 \%$ effective and safe ${ }^{8-10}$. In addition, inactivated vaccines or viral vector vaccines have also been available in certain countries and areas $5,7,9,10$. For example, the adenovirus-vector-based vaccine (ChAdOx1 nCoV-19/AZD1222) has reportedly achieved $62 \%$ efficacy in initial trials ${ }^{11}$. The phase 3 reports of another adenovirus-based vaccine (Ad26.COV2.S) has indicated $85 \%$ efficacy against severe disease or death ${ }^{12,13}$.

However, the recent emergence of various SARS-CoV-2 variants with mutations in the spike region is raising concerns about the efficacy of vaccines. The D614G and B.1.1.7 (alpha/N501Y) variants appear to be without antigenic escape ${ }^{14,15}$. However, the B.1.351 (beta) variant is reportedly represents a neutralization escape variant to convalescent sera ${ }^{16}$. The phase 3 results of NVX-CoV2373 (a 
nanoparticle, protein-based vaccine) from the United Kingdom indicated $89 \%$ efficacy with over $50 \%$ of cases attributable to the more transmissible alpha variant ${ }^{17}$. However, a phase $2 b$ trial in South Africa showed $60 \%$ efficacy, in which approximately $90 \%$ of the endpoints occurred in subjects infected with the beta variant 18,19 , suggesting that the beta variant is less susceptible to antibodies elicited with the original Wuhan strain antigens, which is in the composition of all the vaccines currently being evaluated

7. Another recent concern is the emergence of a B.1.617.2 (delta) variant, which was first detected in India, is now spreading around the world. This variant of concern (VOC) seems to have less susceptibility to vaccine-elicited protection and increased transmissibility beyond alpha strains ${ }^{20}$.

In the present study, we examined neutralizing activity and S1-binding-antibody response in BNT162b2vaccinated health care workers $(n=225)$ in Japan. We also investigated the correlation among neutralizing activity levels, S1-binding-IgG and -IgM levels, genders, and adverse events. Decline of BNT162b2-elicited immune response and activity of the elite and moderate responders against VOCs were also investigated.

\section{Results}

\section{Demographic characteristics and immune response in the participants.}

We obtained blood samples for antibody testing from a total of 225, 220, 211, and 210 vaccine recipients on days $7,28,60$, and 90 post- 1 st shot, respectively (Table 1). Demographic characteristics of the participants are shown in Table 1. As of the time of enrollment, the average age of the participants was 41.8 years (range: 21 to 72 years), and $69.8 \%$ of the participants were female serving as a physician, nurse, paramedical staff, or administrative staff. None of the participants was in the immunodeficient state or was receiving immunosuppressants or steroids.

Table 1: Study protocol and demographic characteristics of the participants.

We first determined the neutralizing activity against SARS-CoV-2 in serum samples taken on day 7 post1 st shot from 225 participants; however, none of the samples showed detectable neutralization activity $\left(\mathrm{NT}_{50}<20\right.$-fold). We then determined the neutralizing activity in samples taken on day 28 post- 1 st shot from 220 vaccinated participants. As shown in Fig. $1 \mathrm{~A}, \mathrm{NT}_{50}$ levels were substantially diverse among the participants: the geometric mean of $\mathrm{NT}_{50}$ values was 375.2 (range 25.6-2680.0). Very low or no correlation of the $\mathrm{NT}_{50}$ values with ages was identified (Fig. 1A: Spearman's $\rho=-0.22 ; 95 \% \mathrm{Cl}-0.34$ to -0.09). We also examined the levels of S1-binding-lgG and -IgM levels using the HISCL system that enables quantitative and highly sensitive determination of S1-binding-lgG and -lgM levels ${ }^{21}$. The geometric mean of S1-binding-IgG values was 527.0 (range 44.6-3212.2), while that of S1-binding-IgM was 85.1 (range 10.3-1406.5). There was a high positive correlation of the $\mathrm{NT}_{50}$ values with S1-bindingIgG levels (Spearman's $\rho=0.71 ; 95 \% \mathrm{Cl} 0.63$ to 0.77 ) as examined on day 28 post- 1 st shot, while there was only a low positive correlation of the $\mathrm{NT}_{50}$ values with S1-binding-IgM levels (Spearman's $\rho=0.43$; $95 \% \mathrm{Cl} 0.31$ to 0.53 ), suggesting that the neutralizing activity largely resides in IgG fraction of the serum 


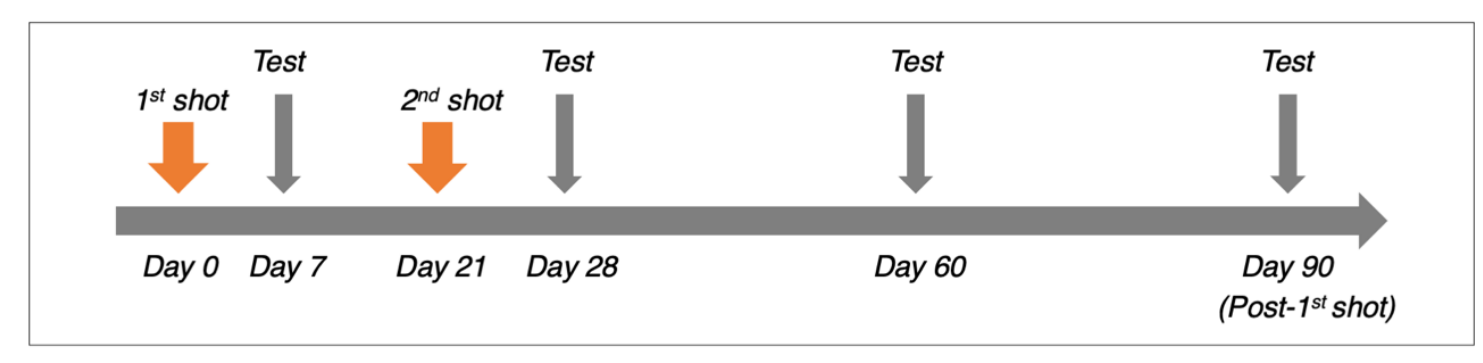

\begin{tabular}{|c|c|c|c|c|c|c|c|}
\hline & & $\begin{array}{l}\text { Day7 } \\
\text { (Post } 1^{\text {st }} \text { dose) }\end{array}$ & (\%) & Day28 & Day60 & Day90 & $\begin{array}{l}\text { post- } 1 \text { st shot, the } \\
\text { correlations of } \mathrm{NT}_{50} \\
\text { levels with both } \operatorname{lgG}\end{array}$ \\
\hline All & & 225 & & 220 & 211 & 210 & and IgM levels \\
\hline \multirow[t]{4}{*}{ Age } & $20-39$ & 97 & 43.1 & 92 & 84 & 84 & low (Spearman's $\rho=$ \\
\hline & $40-59$ & 110 & 48.9 & 110 & 109 & 108 & 0.56 and 0.32 \\
\hline & $\geq 60$ & 18 & 8.0 & 18 & 18 & 18 & respectively) \\
\hline & (Average) & (41.8 y.o.) & & & & & 1). \\
\hline \multirow[t]{2}{*}{ Gender } & Men & 68 & 30.2 & 68 & 63 & 61 & The occurrence of \\
\hline & Women & 157 & 69.8 & 52 & 148 & 149 & adverse effects has \\
\hline \multirow[t]{3}{*}{ Job } & Physicians & 36 & 16.0 & & & & no association with \\
\hline & Nurses & 125 & 55.6 & & & & $\begin{array}{l}\text { the BNT162b2-elicited } \\
\text { neutralizing activity }\end{array}$ \\
\hline & Others & 64 & 28.4 & & & & levels. \\
\hline
\end{tabular}

Commonly observed adverse events reported following BNT162b2 vaccination include injection-site pain, systemic fever, headache, and fatigue ${ }^{10}$. In the present study, the events were observed largely more often following the 2nd shot (Supplementary Fig. 2) as previously reported by Polack et al. ${ }^{10}$ Pains in the inject-site were reported by 67.6 and $61.6 \%$ of the participants and systemic fever $\left(\geq 37.1^{\circ} \mathrm{C}\right)$ was reported by 3.6 and $46.4 \%$ of the participants following the 1 st and 2 nd shots, respectively. Since the severity of pains can be relatively more quantitatively rated than that of other adverse effects such as headache and fatigue, the possible correlate of the $\mathrm{NT}_{50}$ values with the severity of pains rated with the short form McGill pain questionnaire ${ }^{22}$ was first examined. No correlation was seen between the $\mathrm{NT}_{50}$ values and the pain grades assessed following the 2 nd shot (Spearman's $\rho=0.14 ; 95 \% \mathrm{Cl} 0.00$ to 0.26 ). The correlation was also negligible between the $\mathrm{NT}_{50}$ values and the incidence of systemic fever (Spearman's $\rho=0.26 ; 95 \% \mathrm{Cl}$ 0.13 to 0.38 )(Figs. $2 \mathrm{~A}$ and $2 \mathrm{~B}$ ).

The average half-life of neutralizing activity in the vaccinees is approximately 67.8 days and the average time-length for their serums to lose the detectable activity is 198.3 days. 
Considering that recent multiple clinical studies strongly suggest that the presence of high-level neutralizing antibodies is generally sufficient to confer protection against SARS-CoV-2 infection and that the protection against COVID-19 development is largely explained by robust SARS-CoV-2-neutralizing antibody response ${ }^{8-10}$. If so, the once-established neutralizing antibody levels will decrease in time. We thus examined at what rate the levels of $\mathrm{NT}_{50}$ and $\mathrm{S} 1$-bindng-IgG and - IgM levels change by determining those levels from the data on day $28(n=220)$, day $60(n=211)$, and day $90(n=210)$ post- 1 st shot (Figs. $3 \mathrm{~A}-\mathrm{C}$ ). The reduction of all $\mathrm{NT}_{50}$, IgG, and IgM levels from day 28 through day 90 post- 1 st shot was found to occur virtually linearly. By computation, the predicted average half-life of all the $\mathrm{NT}_{50}$ values turned out to be 67.8 days and those of S1-binding-IgG and IgM levels were 53.5 days and 43.6 days, respectively (Fig. 3D). The half-life of the $\mathrm{NT}_{50}$ values and that of S1-binding-IgG were reasonably comparable, corroborating that the neutralizing activity largely resides in the S1-binding IgG fraction. Based on the chronologically linear nature of the reduction identified, we attempted to extrapolate from such half-life values and tried to predict the average time-length for the serums of the participants having significant $\mathrm{NT}_{50}$ values to lose the activity down to under the undetectable level (UDL)(<20-fold)(Fig. 3D). The predicted average time-length for the serums to lose the activity was computated to be 198.3 days, while that of the top $10 \%$ participants to lose the activity was 204.3 days. The time-length of the middle $10 \%$ (between the top $45 \%$ and $55 \%$ ) participants to lose the activity was 187.6 days. For all participants, it was predicted that day 160 after the 1 st shot was when the $80 \%$ lower limit of predicted $\mathrm{NT}_{50}$ levels drops under the detection level (UDL), while day 237 after the 1st shot was when the 80\% upper limit of predicted $\mathrm{NT}_{50}$ levels drops below UDL. Similarly, for the top $10 \%$ participants, the estimated days dropping below UDL were 171 and 243 for lower and upper limits, respectively, while for the middle $10 \%$, the estimated days were 155 and 224, respectively. As for IgG, the predicted time-length for the serums to get undetected was 346.8 days, and days reaching below UDL were 333 and 362 for lower and upper limits, respectively. Likewise, the estimated day reaching below UDL for IgM was 183.6, and the lower and upper limits were 170 and 198 days, respectively (Fig. 3D). We also asked whether the chronological decay rate of neutralization titers and S1-binding-lgG and -IgM differs among three age subgroups: (i) 20-39 yo, (ii) 40-59 yo, and (iii) 60's and beyond. No significant difference was identified among the three age subgroups in the levels of neutralizing titers, $\lg \mathrm{G}$, or $\operatorname{lgM}$ levels $(p=0.60,0.16$, and 0.11 , respectively: Supplementary Figs. $3 A-C$ ). The present data suggest that vaccinated individuals with good neutralization response would lose BNT162b2's protection in 6 to 7 months without regard to age subgroups unless such people achieve robust immune boost response upon the future exposure to SARSCoV-2. Otherwise, they should be protected by another booster vaccine shot or by other protective means.

\section{Neutralization titers, S1-binding-IgG levels, and pain scores in the injection site were greater in women than in men.}

We then asked whether there were differences between genders in neutralization activity levels, S1bindng-IgG and -IgM levels, injection-site pain scores, and systemic fever grades. Statistically significant differences were identified in the levels of neutralization determined on 60 and 90 days post- 1 st shot $(p=$ 0.002 and 0.002 , respectively), S1-binding-IgG levels determined on 28,60 , and 90 days $(p<0.001, p=$ 
0.001 , and $p=<0.001$, respectively) post-1 st -shot, and S1-binding-lgM levels on 60 and 90 days post-1st shot ( $p=0.025$ and 0.044 , respectively)(Supplementary Figs. 4A-C). The injection-site pain score was greater in women $(p<0.001)$ (Supplementary Fig. 4D). However, there was no difference in systemic fever grades between genders (Supplementary Fig. 4E). However, no difference was seen in the decline rates of neutralization activity, S1-binding-lgG and -IgM levels between men and women (Supplementary Fig. 5).

\section{Some serums retain potent activity against various VOCs, but others showed substantially less potent or undetectable activity.}

We finally asked whether the neutralizing antibodies elicited by BNT162b2 vaccination blocked the infectivity and replication of various variants of concerns (VOCs). To this end, we employed serum samples from 6 elite responders ( $\mathrm{NT}_{50}$ values $>1,500$-fold: the top $4 \%$ of all participants' $\mathrm{NT}_{50}$ values as determined on 28 days post- 1 st dose) and serum samples from twelve randomly-selected moderate responders ( $\mathrm{NT}_{50}$ values $=200 \sim$ 1,500-fold) and tested them for their inhibition of the infectivity and cytopathic effect of each variant in the VeroE6 TMPRSS2 $_{2}$ cell-based assay ${ }^{23}$. As shown in Fig. 4, serums from the elite responders $(n=6)$ showed potent inhibition against SARS-CoV-2 ${ }_{05-2 N}$ (Wuhan strain, PANGO lineage B), while they showed less activity against SARS-CoV-2 $2_{\mathrm{QHN} 001}$ and SARS-CoV-2 $2_{\mathrm{QKO02}}$ (alpha), SARS-CoV- ${ }_{5356}$ (kappa), SARS-CoV- ${ }_{1734}$ (delta), and SARS-CoV- 2 TY8- 612 (beta). Serums from moderate responders $(n=12)$ exerted less activity against the Wuhan strain than those from the elite responders. Some serums from the moderate responders also showed substantially low potency to all the VOCs tested. Notably, three serums from the moderate responders showed only marginal activity against SARS-CoV-2 $\mathrm{TY8}-612$ (beta). Two of those three samples had no detectable inhibitory activity against SARS-CoV-2 ${ }_{\text {TY8 }-612}$ (Fig. 4).

\section{Discussion}

In this prospective observational study, 225 healthy individuals [physicians $(n=36)$, nurses $(n=125)$, and other healthcare professionals $(n=64)$ ], who received two doses of $30 \mu \mathrm{g}$ BNT162b2 (Pfizer-BioNTech) vaccine in February 2021, were enrolled, and the correlates of neutralization activity represented by $50 \%$ neutralization titers $\left(\mathrm{NT}_{50}\right.$ ) determined by employing the target living VeroE6 ${ }_{\text {TMPRSS2 }}$ cells and live SARSCoV-2 with ages, adverse effects (AEs) that occur often such as injection-site pain and systemic fever were examined. The kinetics of $\mathrm{NT}_{50}$ values and $\mathrm{S} 1$-binding antibody levels were also examined. There was a significant rise in the $\mathrm{NT}_{50}$ values as determined on day 28 post-1st shot (a week after post $2 \mathrm{nd}$ shot) compared to those on day 7 post- 1 st shot. Correlation was negligible between $\mathrm{NT}_{50}$ values and ages or systemic fever grades. In this regard, most adverse effects that occur within 1-3 days following vaccine shots are thought to be caused by the release of certain pyrogenic and inflammatory cytokines (e.g., interleukin-1, interleukin-6, and tumor-necrosis factor) from antigen-presenting cells (APCs) such as macrophages and dendritic cells when they ingest and process the exogenous antigens (i.e., SARS-CoV-2 spike antigens) and transmit the antigenic information to relevant immune cells. Such early-phase defense events include response to antigenic determinants irrelevant to neutralizing activity but those 
eliciting S1-binding antibodies. The release of the pyrogenic and inflammatory cytokines mostly subsides within days following the vaccine shot. The released cytokines activate the antigen-specific-antibodyproducing B-cells, which respond to the processed antigenic determinants presented by the APCs and start to produce antigen-specific antibodies such as neutralizing antibodies as well as non-neutralizing but S1-binding antibodies. Such antigen-specifically-activated and antibody-producing B-cells continue to produce antibodies. In the case of BNT162b2 vaccination, it appears that it takes 10 to 12 days from the 1 st vaccine shot for the vaccinated individuals to achieve the amounts of neutralizing antibodies that are enough to block the infection of substantial numbers of the virally-targeted cells and to inhibit further spread of the infection ${ }^{9,10}$. It is thought that the release of pyrogenic and inflammatory cytokines and the build-up of the protective antibody levels are different events, occurring chronologically 10-12 days apart. These two different events appear to have resulted in the absence of significant correlates between $\mathrm{NT}_{50}$ levels and $\mathrm{AEs}$ examined in the present study.

In the present study, the $\mathrm{NT}_{50}$ values had a substantial correlation with S1-binding-lgG levels but had only moderate correlation with S1-binding IgM levels, suggesting that the major neutralizing activity resides within the S1-binding IgG fraction. Interestingly, the approximate half-life of $\mathrm{NT}_{50}$ values (67.8 days) and that of S1-binding-IgG levels (53.5 days) were reasonably close to each other, corroborating the assumption of the presence of the major fraction of neutralizing antibodies within IgG fraction. In human body, IgG has concentration-dependent half-life of approximately 21 days and $\operatorname{lgM}$ around 5-6 days ${ }^{24}$. By contrast, the half-lifes of $\mathrm{NT}_{50}, \mathrm{S1}$-bindng-IgG, and -IgM levels determined in the present study were much longer with 43.6-67.8 days. This discrepancy is perhaps attributed to the persistence of continuously-antibody-producing B cells over weeks or months in the body of the participants following the vaccination ${ }^{25,26}$, thereby the half-lifes of neutralizing activity and S1-binding-IgG and -IgM levels have been extended as compared to the physiological half-lifes of IgG and IgM. Of note, the assumption of the half-lifes in the present work was based on the chronologically linear nature of the reduction observed during the present study period (Fig. 3D). Recently, Doria-Rose et al. reported that anti-SARS-CoV-2 antibodies persist through 6 months after the second dose of mRNA-1273 administration ${ }^{27}$. However, the study was of a relatively small scale $(n=33)$, and more definite data are needed for constructing more protective measures.

There is a growing body of evidence that COVID-19 results in more severe symptoms and greater mortality among men than among women ${ }^{28,29}$. A cohort study of 17 million adults in England has revealed a strong association between male sex and the risk of death from COVID-19 (hazard ratio 1.59, $95 \%$ confidence interval $1.53-1.65)^{30}$. In the present data set, significantly greater levels of $\mathrm{NT}_{50}, \mathrm{~S} 1$ binding-IgG and -IgM were documented in women than in men when examined on 28,60, and 90 days post-1st shot, while there was no difference in either of $\mathrm{NT}_{50}$, S1-binding-lgG or -lgM levels on day 7 post1 st dose (Supplementary Figs. 4A-C). These results apparently relate to the findings by others reporting that women, in general, have more robust ability to control infectious pathogens (i.e., SARS-CoV-2) than men ${ }^{30,31}$. Indeed, there is increasing evidence indicating strong correlation between SARS-CoV-2- 
neuralizing antibody titers and clinical efficacy, suggesting that a neutralizing antibody response provides the primary contribution to protection against COVID-19 ${ }^{32}$ and that the presence of high levels of neutralizing antibody is largely sufficient for protection against SARS-CoV-2 infection and clinical onset upon exposure to the virus ${ }^{33,34}$. In fact, Imai et al. have reported that the administration of convalescent plasma from previously-SARS-CoV-2-infected hamsters completely protected newly SARS-CoV-2-exposed hamsters from contracting viral pneumonitis ${ }^{35}$. Thus, the greater neutralizing activity in women than in men observed in the present study can contribute at least in part to the gender differences in COVID-19 disease outcomes.

We also examined how the BNT162b2-elicited neutralizing antibodies blocked the infectivity and cytopathic effect of five different variants of concerns in the cell-based assays using various infectious variants (one Wuhan strain, two alpha strains, one strain each of beta, delta and kappa strains). Six selected serums from elite responders showed quite potent activity to the alpha, kappa, and delta variants, while they exerted relatively moderate activity against the beta strain (Fig. 4). On the other hand, one of the randomly-selected 12 serums from moderate responders showed a marginal activity $\left(\mathrm{NT}_{50}\right.$ value of 40 -fold) and two of them failed to show detectable activity ( $\mathrm{NT}_{50}$ values $<20$-fold) against the beta strain (Fig. 4). These data suggest that BNT162b2-receiving vaccinees who develop high magnitudes of neutralizing antibody should probably be well protected against the infection by most variants; however, those who develop only low levels of neutralizing antibody may be vulnerable to the infection by certain variants such as beta strains. If so, to such low-responders to BNT162b2 even after the $2 \mathrm{nd}$ shot, an additional 3rd shot may be needed. If the 3rd dose of the same vaccine fails to elicit good levels of neutralizing antibodies, new types of vaccines with different platform have to be stratified.

\section{Methods}

\section{Participants and serum specimens.}

Serum samples were collected from 225 vaccinated health care workers at JCHO Kumamoto General Hospital (Kumamoto, Japan). All the 225 participants were of Japanese citizen. Serum samples were analyzed at the National Center for Global Health and Medicine (NCGM) in Tokyo. The Ethics Committees from the Kumamoto General Hospital and NCGM approved this study (Kumamoto General Hospital No. 180, and NCGM-G-004176-00, respectively). Each participant provided a written informed consent, and this study abided by the Declaration of Helsinki principles. The vaccination (on days 0 and 21) and serum collection (from day 7 through day 90 post-1 st shot) were conducted as shown in Table 1.

\section{Cells and viruses.}

VeroE6 ${ }_{\text {TMPRSS2 }}$ cells ${ }^{23}$ were obtained from Japanese Collection of Research Bioresources (JCRB) Cell Bank (Osaka, Japan). VeroE6 TMPRSS2 $_{2}$ cells were maintained in DMEM supplemented with $10 \%$ FCS, 100 $\mu \mathrm{g} / \mathrm{ml}$ of penicillin, $100 \mu \mathrm{g} / \mathrm{ml}$ of streptomycin, and $1 \mathrm{mg} / \mathrm{mL}$ of G418. SARS-CoV-2 NCGM-05-2N strain (SARS-CoV-2 $05-2 \mathrm{~N}$ ) was isolated from nasopharyngeal swabs of a patient with COVID-19, who was 
admitted to the NCGM hospital ${ }^{36,37}$. Five clinically isolated SARS-CoV-2 mutant strains were used in the current study: two B.1.1.7 (alpha) strains [hCoV-19/Japan/QHN001/2020 (SARS-CoV-2 QHN001, GISAID Accession ID; EPI_ISL_804007) and hCoV-19/Japan/QK002/2020 (SARS-CoV-2 ${ }_{\text {QK002, }}$ G ISAID Accession

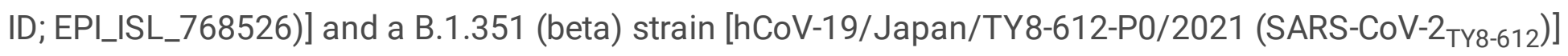
were obtained from National Institute of Infectious Diseases, Tokyo, Japan. A B.1.617.1 (kappa) strain [TKYTK5356_2021 (SARS-CoV-2 5356, DDBJ Accession ID; LC633761)] and a B.1.617.2 (delta) strain [hCoV-19/Japan/TKYK01734/2021 (SARS-CoV-2 ${ }_{1734}$, GISAID Accession ID; EPI_ISL_2080609)] were provided from Tokyo Metropolitan Institute of public Health, Tokyo, Japan. Each variant was confirmed to contain each VOC-specific amino acid substitutions before the assays conducted in the present study (vide infra).

\section{Neutralization assay.}

The neutralizing activity of serums from vaccinated individuals was determined by quantifying the serum-mediated suppression of the cytopathic effect (CPE) of each SARS-CoV-2 strain in VeroE6 ${ }_{\text {TMPRSS2 }}$ cells as previously described with minor modifications ${ }^{36}$. In brief, each serum was 4 -fold serially diluted in culture medium. The diluted sera were incubated with $10050 \%$ tissue culture infectious dose $\left(\mathrm{TCID}_{50}\right)$ of viruses at $37^{\circ} \mathrm{C}$ for $20 \mathrm{~min}$ (final serum dilution range of 1:20 to 1:4000), after which the serum-virus mixtures were inoculated to VeroE6 ${ }_{\text {TMPRSS2 }}$ cells $\left(1.0 \times 10^{4} /\right.$ well) in 96-well plates. For SARS-CoV-2 strains used in this assay are as follows: a wild-type strain, SARS-CoV-2 $05-2 \mathrm{~N}$ (PANGO lineage B) ${ }^{36,37}$, two alpha variants (SARS-CoV-2 ${ }_{\mathrm{QHN} 001}$ and SARS-CoV-2 ${ }_{\mathrm{QK} 002}$ ), a beta variant SARS-CoV- $2_{\mathrm{TY} 8-612}$, a delta variant SARS-CoV- ${ }_{1734}$, and a kappa variant SARS-CoV- $2_{5336}$. After culturing the cells for 3 days, the levels of CPE observed in SARS-CoV-2-exposed cells were determined using the WST-8 assay employing Cell Counting Kit-8 (Dojindo, Kumamoto, Japan). The serum dilution that gave 50\% inhibition of CPE was defined as the $50 \%$ neutralization titer ( $\mathrm{NT}_{50}$ ). Each serum was tested in duplicate.

\section{Measurement of anti-SARS-CoV-2 antibody titers.}

Measurement of 3 anti-SARS-CoV-2 antibody levels (anti-S1-IgG, anti-S1-IgM, and anti-N-IgG) in each participant was performed using the chemiluminescence enzyme immunoassay (CLEIA) platform (HISCL) manufactured by Sysmex Co. (Kobe, Japan) as previously reported ${ }^{21}$.

\section{Statistical analyses.}

Out of the 225 participants, one participant, who had been infected by SARS-CoV-2 with PCR positivity documented was primarily excluded. Demographic characteristics of the participants are described in Table 1. Correlates of neutralizing activity levels with S1-binding-lgG and -IgM levels, ages, genders, pain scores in the injection-site, and systemic fever up to $40^{\circ} \mathrm{C}$ were examined by Spearman rank correlation coefficient. Also, neutralizing activity levels, S1-binding-lgG, and -lgM, pain scores, systemic fever were compared between genders using the Wilcoxon rank sum test. As for participants with normal fever, their 
temperature was treated as 36.89 degree, a normal body temperature in Japanese ${ }^{38}$. Percentage of the adverse events reported in writing following the $1 \mathrm{st}$ and 2 nd dose administration were determined and assessed in regard to gender. The differences in neutralization activity between each measurement time point were tested by the Wilcoxon rank sum test, and were assessed among categorized age subgroups. Similarly, difference of S1-binding-IgG and -lgM levels among time points were tested. Decline of neutralizing activity over 90 days post- 1 st shot was assessed using the mixed-effects model including time as a main effect and intercept as a random effect. Also, the prediction slope and its $80 \%$ prediction interval were generated by drawing a sampling distribution for the fixed effects and then estimating the fitted value across that distribution. The merTools package (version 0.5.2) in R software was used for prediction. The decline for S1-binding-lgG and -lgM levels was assessed similarly. All the analyses were conducted with the use of R, version 4.1.0 (R Foundation for Statistical Computing).

\section{Declarations}

\section{Acknowledgments}

The authors thank Drs. Kenji Sadamasu, Mami Nagashima, Hiroyuki Asakura, and Mr. Isao Yoshida for providing two SARS-CoV-2 variants, Drs. Nobuyo Higashi-Kuwata, Shinichiro Hattori, Kouki Matsuda, Ms. Mariko Kato and Ms. Sachiko Otsu for helping some experiments, and Dr. Norihiro Kokudo for critical discussion for the manuscript. This research was supported in part by a grant from the Japan Agency for Medical Research and Development to Maeda (grant number JP20fk0108260, JP20fk0108502) and to Mitsuya (grant number 20fk0108502), and in part by a grant for MHLW Research on Emerging and Reemerging Infectious Diseases and Immunization Program to Maeda (grant number JPMH20HA1006) from Ministry of Health, Labor and Welfare, and in part by a grant for COVID-19 to Mitsuya (grant number 19A3001) and Maeda (grant number 20A2003D) from the Intramural Research Program of National Center for Global Health and Medicine, and in part by the Intramural Research Program of the Center for Cancer Research, National Cancer Institute, National Institutes of Health (Mitsuya).

\section{Author Contributions}

$\mathrm{KM}$ and $\mathrm{HM}$ had access to all data in this study and took and hold all responsibility for the integrity of the data and the accuracy of the data analysis. KM and HM: Concept and design. KM, MA, KT, TM, KN, YT, HG, KY, SO, TS, and TY: Acquisition, analysis, or interpretation of data. YU, YS, AF, and HM: Statistical analysis. AF, YI, HN, MK, SM, AM, WS, and SS: Administrative, technical, or material support. $\mathrm{KM}$ and $\mathrm{HM}$ : Original draft writing. All authors: Reading and writing manuscript.

\section{Completing Interests}

Matsushima, Noda, Sato, and Yoshida are employees of Sysmex Corporation. Other authors declare that there is no conflict of interest.

\section{References}


1. World Health Organization. Coronavirus disease (COVID-19) outbreak situation. https://www.who.int/publications/m/item/weekly-epidemiological-update-on-covid-19--29-june2021. (2021).

2. Wu, F. et al. A new coronavirus associated with human respiratory disease in China. Nature, $\mathbf{5 7 9}$, 265-269 (2020).

3. Zhou, P. et al. A pneumonia outbreak associated with a new coronavirus of probable bat origin. Nature, 579, 270-273 (2020).

4. Mitsuya, H. \& Kokudo, N. Sustaining containment of COVID-19: global sharing for pandemic response. Glob Health Med, 2, 53-55 (2020).

5. Dal-Re, R. et al. Ongoing and future COVID-19 vaccine clinical trials: challenges and opportunities. Lancet Infect Dis(2021).

6. Cohen, J. Effective vaccine offers shot of hope for pandemic., 370, 748-749 (2020).

7. Richman, D. D. COVID-19 vaccines: implementation, limitations and opportunities. Glob Health Med, 3, 1-5 (2021).

8. Pfizer, N. / Pfizer and BioNTech Conclude Phase 3 Study of COVID-19 Vaccine Candidate, Meeting All Primary Efficacy Endpoints. https://www.pfizer.com/news/press-release/press-release-detail/pfizerand-biontech-conclude-phase-3-study-covid-19-vaccine. (2021).

9. Baden, L. R. et al. Efficacy and Safety of the mRNA-1273 SARS-CoV-2 Vaccine. N Engl J Med, 384, 403-416 (2021).

10. Polack, F. P. et al. Safety and Efficacy of the BNT162b2 mRNA Covid-19 Vaccine. N Engl J Med, 383, 2603-2615 (2020).

11. Voysey, M. et al. Safety and efficacy of the ChAdOx1 nCoV-19 vaccine (AZD1222) against SARS-CoV2: an interim analysis of four randomised controlled trials in Brazil, South Africa, and the UK., 397, 99-111 (2021).

12. Johnson \& Johnson, Johnson \& Johnson COVID-19 Vaccine Authorized by U.S. FDA For Emergency Use. \& - First Single-Shot Vaccine in Fight Against Global Pandemic. https://www.jnj.com/johnsonjohnson-covid-19-vaccine-authorized-by-u-s-fda-for-emergency-usefirst-single-shot-vaccine-in-fightagainst-global-pandemic. (2021).

13. Sadoff, J. et al. Safety and Efficacy of Single-Dose Ad26.COV2.S Vaccine against Covid-19. N Engl J Med, 384, 2187-2201 (2021).

14. Korber, B. et al. Tracking Changes in SARS-CoV-2 Spike: Evidence that D614G Increases Infectivity of the COVID-19 Virus., 182, 812-827819 (2020).

15. Xie, X. et al. Neutralization of SARS-CoV-2 spike 69/70 deletion, E484K and N501Y variants by BNT162b2 vaccine-elicited sera. Nat Med, 27, 620-621 (2021).

16. Cele, S. et al. Escape of SARS-CoV-2 501Y.V2 from neutralization by convalescent plasma. Nature, 593, 142-146 (2021).

17. Heath, P. T. et al. Safety and Efficacy of NVX-CoV2373 Covid-19 Vaccine.N Eng/ J Med(2021). 
18. Novavax \& Novavax COVID-19 Vaccine Demonstrates 89.3\% Efficacy in UK Phase 3 Trial. https://www.globenewswire.com/news-release/2021/01/28/2166253/0/en/Novavax-COVID-19Vaccine-Demonstrates-89-3-Efficacy-in-UK-Phase-3-Trial.html. (2021).

19. Shinde, V. et al. Efficacy of NVX-CoV2373 Covid-19 Vaccine against the B.1.351 Variant. N Engl J Med, 384, 1899-1909 (2021).

20. Wall, E. C. et al. Neutralising antibody activity against SARS-CoV-2 VOCs B.1.617.2 and B.1.351 by BNT162b2 vaccination., 397, 2331-2333 (2021).

21. Noda, K. et al. A novel highly quantitative and reproducible assay for the detection of anti-SARS-CoV2 IgG and IgM antibodies. Sci Rep, 11, 5198 (2021).

22. Melzack, R. The short-form McGill Pain Questionnaire., 30, 191-197 (1987).

23. Matsuyama, S. et al. Enhanced isolation of SARS-CoV-2 by TMPRSS2-expressing cells. Proc Natl Acad Sci U S A, 117, 7001-7003 (2020).

24. Waldmann, T. A. \& Strober, W. Metabolism of immunoglobulins. Prog Allergy, 13, 1-110 (1969).

25. Turner, J. S. et al. SARS-CoV-2 mRNA vaccines induce persistent human germinal centre responses.Nature(2021).

26. Stephens, D. S. \& McElrath, M. J. COVID-19 and the Path to Immunity. JAMA, 324, 1279-1281 (2020).

27. Doria-Rose, N. et al. Antibody Persistence through 6 Months after the Second Dose of mRNA-1273 Vaccine for Covid-19. N Engl J Med, 384, 2259-2261 (2021).

28. Chen, N. et al. Epidemiological and clinical characteristics of 99 cases of 2019 novel coronavirus pneumonia in Wuhan, China: a descriptive study., 395, 507-513 (2020).

29. Yang, X. et al. Clinical course and outcomes of critically ill patients with SARS-CoV-2 pneumonia in Wuhan, China: a single-centered, retrospective, observational study. Lancet Respir Med, 8, 475-481 (2020).

30. Williamson, E. J. et al. Factors associated with COVID-19-related death using OpenSAFELY. Nature, 584, 430-436 (2020).

31. Collazos, J., Asensi, V. \& Carton, J. A. Sex differences in the clinical, immunological and virological parameters of HIV-infected patients treated with HAART. AIDS, 21, 835-843 (2007).

32. Voysey, M. et al. Single-dose administration and the influence of the timing of the booster dose on immunogenicity and efficacy of ChAdOx1 nCoV-19 (AZD1222) vaccine: a pooled analysis of four randomised trials., 397, 881-891 (2021).

33. Khoury, D. et al. What level of neutralising antibody protects from COVID-19? medRxiv(2021).

34. Earle, K. A. et al. Evidence for antibody as a protective correlate for COVID-19 vaccines.medRxiv(2021).

35. Imai, M. et al. Syrian hamsters as a small animal model for SARS-CoV-2 infection and countermeasure development. Proc Natl Acad Sci U S A, 117, 16587-16595 (2020). 
36. Maeda, K. et al. Neutralization of SARS-CoV-2 with IgG from COVID-19-convalescent plasma. Sci Rep, 11, 5563 (2021).

37. Hattori, S. I. et al. GRL-0920, an Indole Chloropyridinyl Ester, Completely Blocks SARS-CoV-2 Infection.mBio11(2020).

38. Wada, Y. For health care by taking the body temperature. The Japan Society of Mechanical Engineers, 112, 484-485 (2009).

39. Takamatsu, Y. et al. Highly-Neutralizing COVID-19-Convalescent-Plasmas Potently Block SARS-CoV-2 Replication and Pneumonia in Syrian Hamsters. bioRxiv(2021).

\section{Figures}
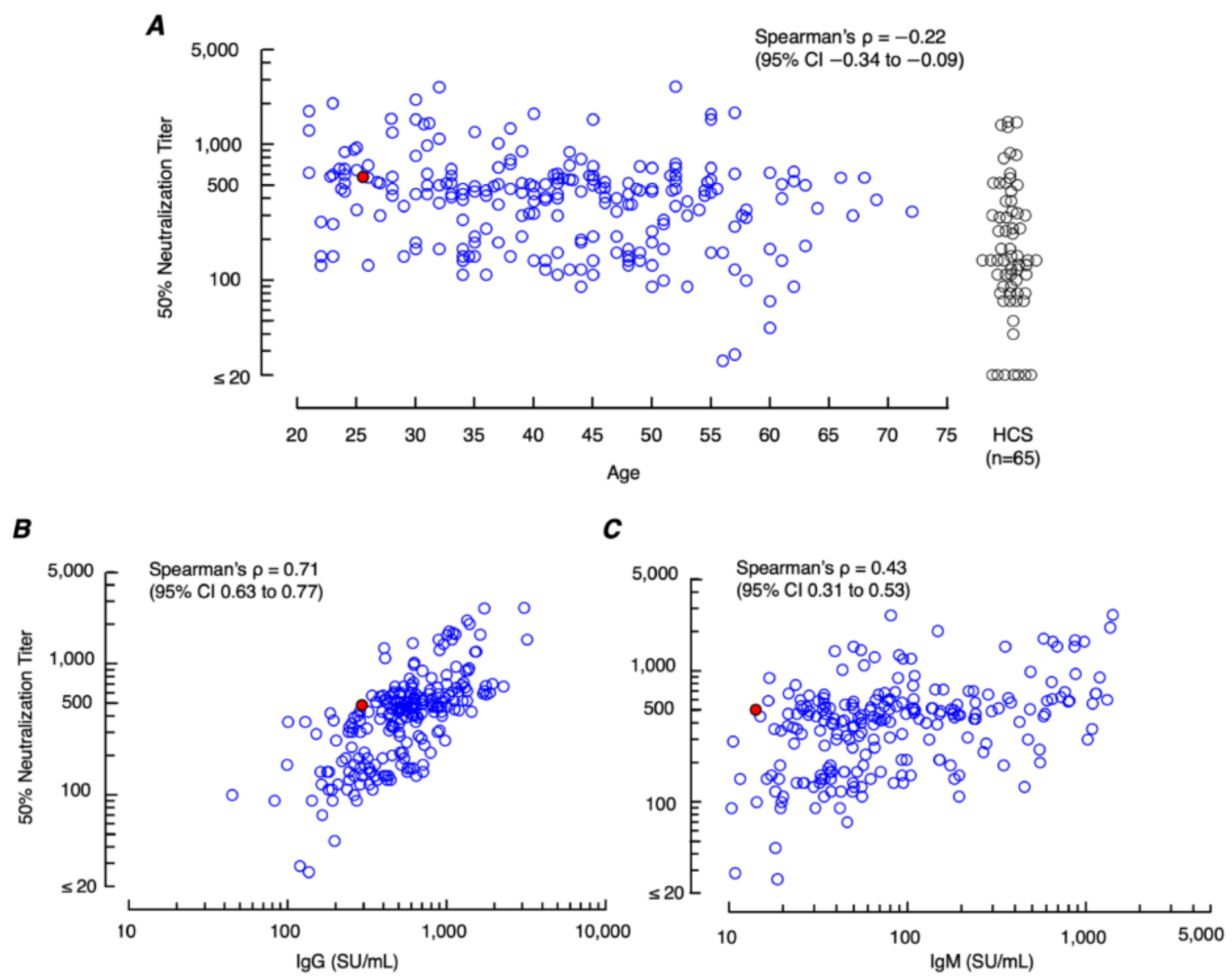

Figure 1 
Correlations of neutralizing titers with ages and S1-binding-lgG and -lgM levels. A. Correlation between neutralizing titers (NT50s) and ages (on day 28 post-1st shot). The age range of the study participants was 21 to 72 (average 41.8 y.o.). A correlation is negligible between NT50 values and ages (Spearman's $\rho=-0.22: 95 \% \mathrm{Cl}-0.34$ to -0.09$)$. The geometric mean NT50 of the values from all participants $(n=225)$ was 375.2 -fold (range 25.6-2,680-fold), greater by a factor of 2.3 than the geometric mean NT50 from 65 COVID-19-convalescent patients (geometric mean=163.0-fold; range 20.0-1470-fold) shown as references on the far right (human COVID-19-convalescent serum: HCS). B. A high correlation is identified (Spearman's $\rho=0.71 ; 95 \% \mathrm{Cl} 0.63$ to 0.77 ) between NT50 values and S1-binding-lgG levels in samples obtained on day 28 post-1st dose. C. Moderate correlation is seen between neutralizing titers and S1binding-lgM levels (Spearman's $\rho=0.43 ; 95 \% \mathrm{Cl} 0.31$ to 0.53 ). One participant, who had been infected with SARS-CoV-2 with PCR-positivity documented, is indicated as a solid-red solitary circle. This participant was excluded from all analyses at later timepoints.
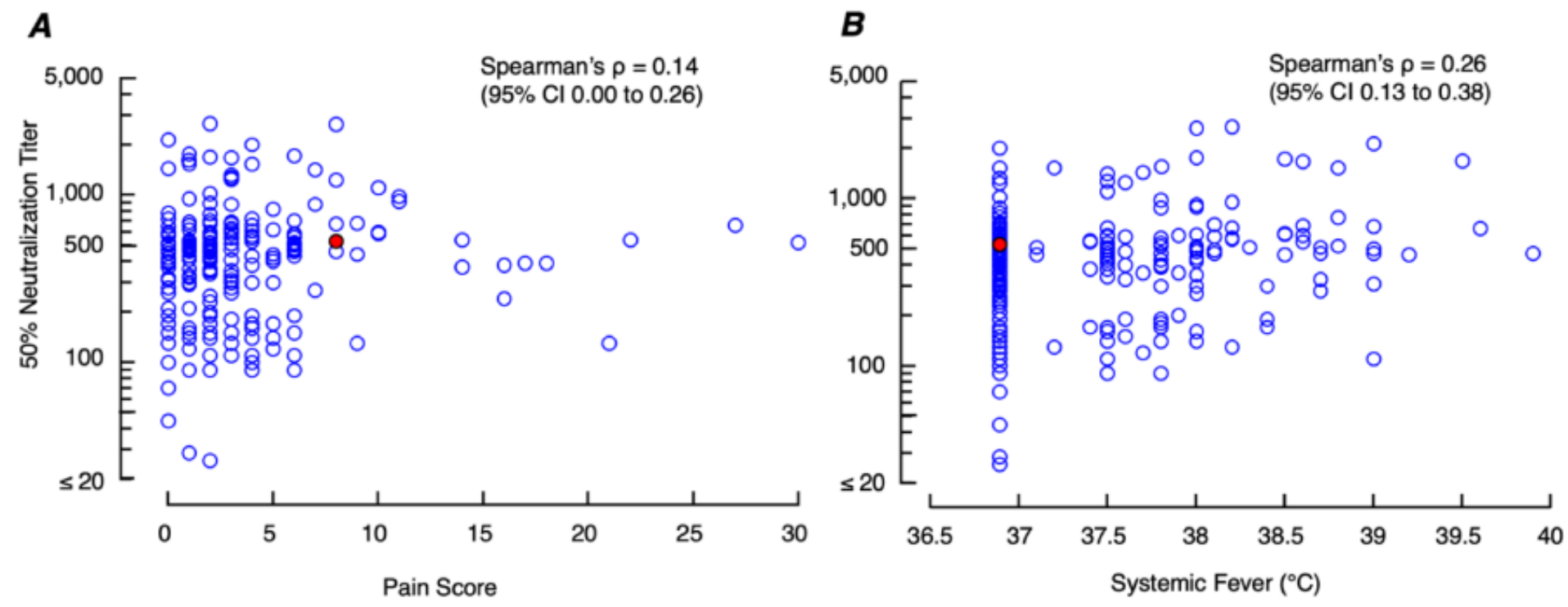

Figure 2

Correlations of neutralizing titers with injection-site pain scores and systemic fever grades. A. No correlation was seen between NT50 values and injection-site pain (Spearman's $\rho=0.14 ; 95 \% \mathrm{Cl} 0.00$ to 0.26). The injection-site pain following the 2 nd BNT162b2 dose was scored by using the short-form McGill Pain Questionnaire22. B. Correlation was negligible between NT50 values and systemic fever grades (Spearman's $\rho=0.26 ; 95 \% \mathrm{Cl} 0.13$ to 0.38 ). A solid-red circle indicates a person with previous SARS-CoV-2 infection documented. 


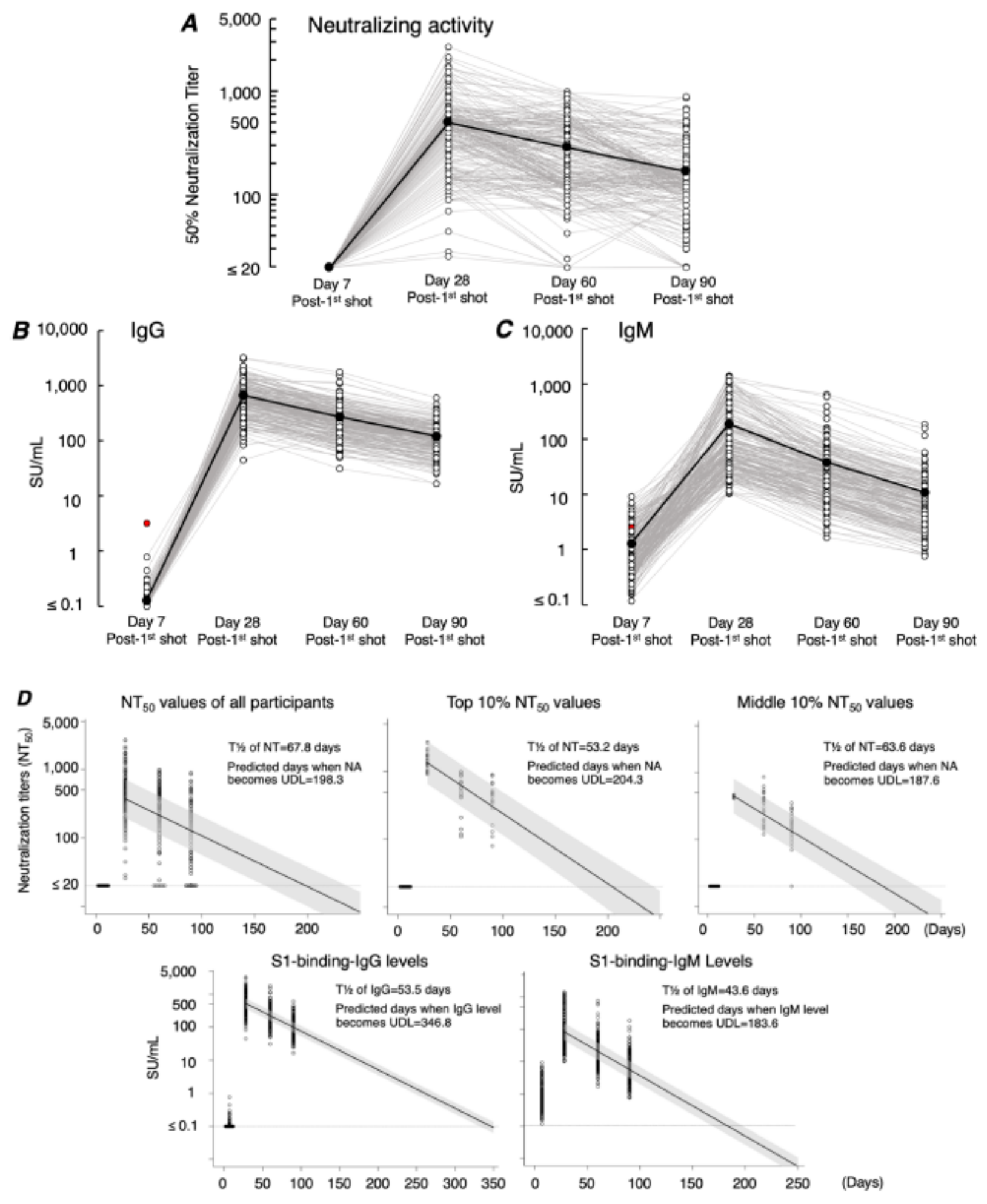

\section{Figure 3}

Kinetics of neutralizing activity and S1-binding-IgG and -IgM levels. Time-course analyses of neutralizing activity for 90 days were conducted. The 1 st vaccine was administered on day 0 , and the 2 nd vaccine on day 21. Blood samples from vaccinated individuals were obtained on days $7,28,60$, and 90 post 1 st shot as illustrated in Table 1. A. Neutralizing activity is shown as NT50 (50\% neutralizing titer). The NT50 value of 20 -fold is the detection limit and values determined to be less than 20 -fold were treated as 20 - 
fold. B and C. Kinetics of S1-binding-IgG and -IgM levels are shown. The average values of each data point are shown in black solid circles, which are connected with solid black lines. One participant, who had been infected with SARS-CoV-2 with PCR-positivity documented, is indicated as a solid-red solitary circle in B and C. This participant was excluded from all analyses at later timepoints. D. Decline of neutralizing activity, S1-binding-lgG and -IgM over 90 days post-1st shot. The solid-black lines consist of predicted values estimated by mixed effects model, and the shaded areas denote corresponding $80 \%$ prediction intervals. The dashed horizontal lines in the upper three panels denote the NT50 detection limit of 20 -fold. NT50 values determined to be less than 20 -fold were treated as 20 -fold. The lowest detection limit for S1-binding-lgG and -IgM quantification shown as dashed horizontal lines in the two lower panels was $0.1 \mathrm{SU} / \mathrm{ml}$ and the values lower than $0.1 \mathrm{SU} / \mathrm{ml}$ were calculated as $0.1 \mathrm{SU} / \mathrm{ml}$.

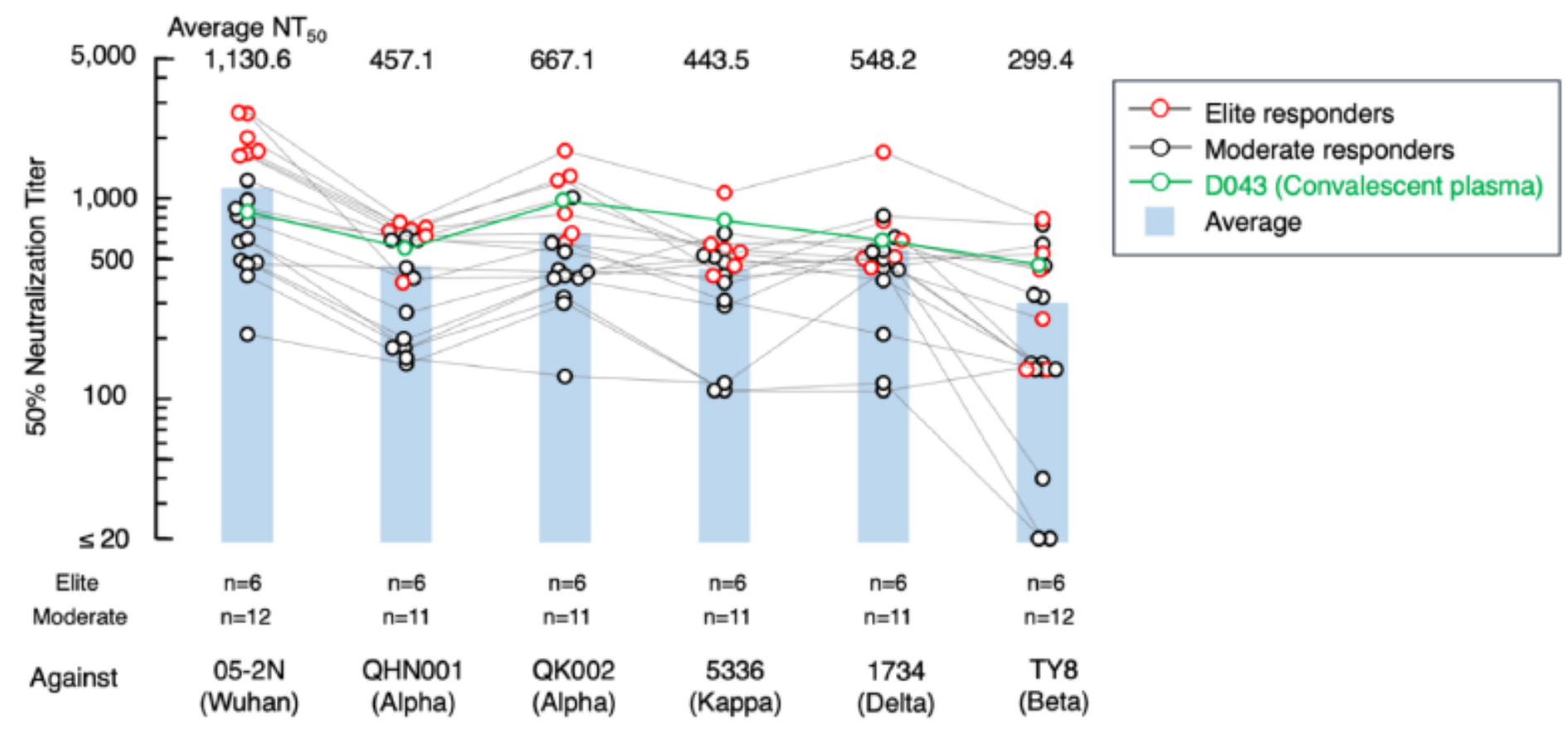

\section{Figure 4}

Blockade of the infectivity and replication of SARS-CoV-2 variants by vaccinees' serums. The activity of vaccinees' serums to block the infectivity and replication of 5 SARS-CoV-2 variants (alpha variants: SARSCoV-2QHN001 and SARS-CoV-2QK002; a beta strain: SARS-CoV-2TY8-612; a delta strain: SARS-CoV21734; and a kappa strain: SARS-CoV-25356) was evaluated. A Wuhan strain SARS-CoV-205-2N 37 was employed as a reference SARS-CoV-2. Six serums were from elite responders (NT50>1,500-fold) and 12 serums were from randomly-selected moderate responders (NT50=200 1,500-fold). The NT50 titers of each serum against 6 SARS-CoV-2 strains are shown in red circles (for 6 elite responders) and in black circles (for 12 moderate responders). D043 is a serum from a COVID-19-convalescent patient 39 and served as an internal control in the assays.

\section{Supplementary Files}


This is a list of supplementary files associated with this preprint. Click to download.

- MaedaetalSupplementaryfigures.pdf 